\title{
Pilot study on virtual imaging for patient information on radiotherapy planning and delivery
}

\author{
J. Sulé-Suso a, b, *, S. Finney a , J. Bisson a, S. Hammersley ${ }^{\text {a }, ~ S . ~ J a s s e l ~}{ }^{\text {a }}$, R. Knight ${ }^{\text {a }}$, C. Hicks ${ }^{\text {a }}$,

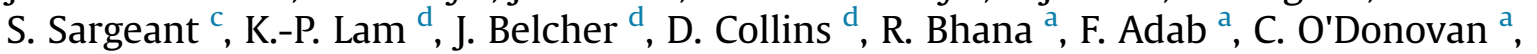 \\ A. Moloney ${ }^{\mathrm{e}}$ \\ ${ }^{a}$ Cancer Centre, City General Hospital, University Hospital of North Staffordshire, Newcastle Rd, Stoke on Trent, Staffordshire ST4 6QG, UK \\ ${ }^{\mathrm{b}}$ Institute for Science and Technology in Medicine, Keele University, Guy Hilton Research Centre, Thornburrow Drive, Stoke on Trent ST4 7QB, UK \\ c Faculty of Health Sciences and Medicine, Bond University, Robina QLD 4229, Australia \\ ${ }^{\mathrm{d}}$ School of Computing and Mathematics, Keele University, Keele ST5 5BG, UK \\ e Physics Department, Cancer Centre, City General Hospital, University Hospital of North Staffordshire, Newcastle Rd, Stoke on Trent, Staffordshire ST4 6QG, \\ $U K$
}

\section{A R T I C L E I N F O}

\section{Article history:}

Received 8 August 2014

Received in revised form

30 January 2015

Accepted 3 February 2015

Available online $\mathrm{xxx}$

\section{Keywords:}

Radiotherapy

Virtual imaging

VERT

Patient information and communication

\begin{abstract}
A B S T R A C T
It is widely accepted that health professionals might sometimes underestimate cancer patients' needs for information on the complex process of radiotherapy (RT) planning and delivery. Furthermore, relatives might also feel excluded from the treatment of their loved ones. This pilot study was carried out in order to assess whether both patients and their relatives would welcome further information on RT planning and delivery using the virtual reality (VR) system VERT. One hundred and fifty patients with different types of cancer receiving radical RT were included in the study. Patients and relatives were shown using VERT on a one-to-one basis with an oncologist or a radiographer, a standard room where RT is given, a linear accelerator, and how RT is planned and delivered using their own planning CT Scans. Patients welcomed this information as it helped them to reduce their fears about RT. Relatives felt also more involved in the treatment of their loved one. The results obtained in this pilot study show that VR aids could become an important tool for delivering information on RT to both patients and relatives.
\end{abstract}

(C) 2015 The College of Radiographers. Published by Elsevier Ltd. All rights reserved.

\section{Introduction}

There is clear evidence suggesting there is often a gap between the information provided to patients regarding their disease and management, and the amount of information patients wish to receive. ${ }^{1}$ Furthermore, it is not uncommon for clinicians to underestimate cancer patients' needs for information, ${ }^{2}$ especially when the vast majority of cancer patients want to be thoroughly informed about their illness. ${ }^{3}$ Despite this, there are still problems with respect to the provision of information which could satisfy most patients. ${ }^{4}$ The importance of the provision of information to patients has been stressed by the Department of Health ${ }^{5}$ stating that is a key requirement in current cancer services standards in the

* Corresponding author. Cancer Centre, City General Hospital, University Hospital of North Staffordshire, Newcastle Rd, Stoke on Trent, Staffordshire ST4 6QG, UK. Tel.: +4401782 672517; fax: +4401782672601.

E-mail address: josep.sulesuso@uhns.nhs.uk (J. Sulé-Suso).
UK. However, it cannot be assumed that all patients want substantial information regarding their treatment as some might prefer to fully trust their doctor rather than being involved in taking any decisions that could affect their management. While several studies have addressed these issues, ${ }^{6,7}$ further work is still required to improve the understanding of patients' needs. Furthermore, relatives might feel sometimes disengaged in the management of their loved ones and might consider themselves being excluded from several areas within the management process. The negative psychological impact upon partners and other relatives of cancer patients is well documented, ${ }^{8}$ and such negative effect is (amongst many other factors) widely associated with lack of medical information given to them. It has long been argued that providing tailored information to relatives as well as patients in general cancer care is a positive aspect of the treatment process ${ }^{9}$ and there is little doubt that such inclusionary practice should be encouraged in cancer care.

A clear example where provision of information to patients is paramount and where often relatives feel excluded is in the case of 
patients with cancer undergoing radiotherapy (RT). Unlike with chemotherapy, relatives cannot be present during RT delivery for obvious reasons of radiation protection. In these cases, oncologists explain to both patients and relatives how RT is planned and given, and the possible side effects using CT Scans as an aid. However, for some patients and their relatives, radiation might be an abstract, non tangible concept compared to, as an example, being able to see a tumour or the different organs in a CT Scan. Also, patients and relatives are often bombarded with components of information regarding their disease and treatment from differing health professionals and at various stages of their pathway. Unfortunately, collating this information to accurately describe the complex process of RT is often difficult, and therefore a virtual reality (VR) system can graphically aid in this process, demonstrating the aims of treatment, the equipment and environment involved, and the critical importance of patient compliance to improve reproducibility and reduce side effects. Another important aspect for these patients and their relatives is the possibility to see the room where they will receive RT before starting treatment. This could improve the delivery of information and both patients and relatives' levels of satisfaction with the service. However, RT departments all over the UK and abroad are busy facilities and, in general, do not have the capacity to provide this service.

Most of these issues could be tackled using a virtual imaging system (in 2D and in 3D) which would show patients and relatives not only how RT is planned and delivered but also the room where treatment is given and the machine delivering it. One technology that could improve the interaction between health professionals and patients in RT planning is the VERT 3D imaging system. ${ }^{10,11}$ The importance of a 3D display in radiation therapy was recognized around 15 years ago. ${ }^{12}$ A full stereoscopic 3D computer model of a Linac was first developed in $2005^{10}$ and the world's first 3D virtual reality training centre integrated in the clinic was established in 2007. ${ }^{13}$ Furthermore, in 2007, the National Radiotherapy Advisory Group (NRAG) proposed the adoption of VR training in RT throughout the United Kingdom. ${ }^{14}$ The VERT 3D imaging system allows patients to see in 3D their own CT Scans, and how RT is planned and delivered. Other potential uses include surgical demonstration and simulation, and teaching. ${ }^{13,15,16}$

To explore how patients might view their experiences of receiving extra information about their treatment we have carried out this pilot study using the VERT 3D imaging system. This system projects 3D images of the treatment room, a linear accelerator (Linac), a treatment couch and various treatment aids (Fig. 1). These

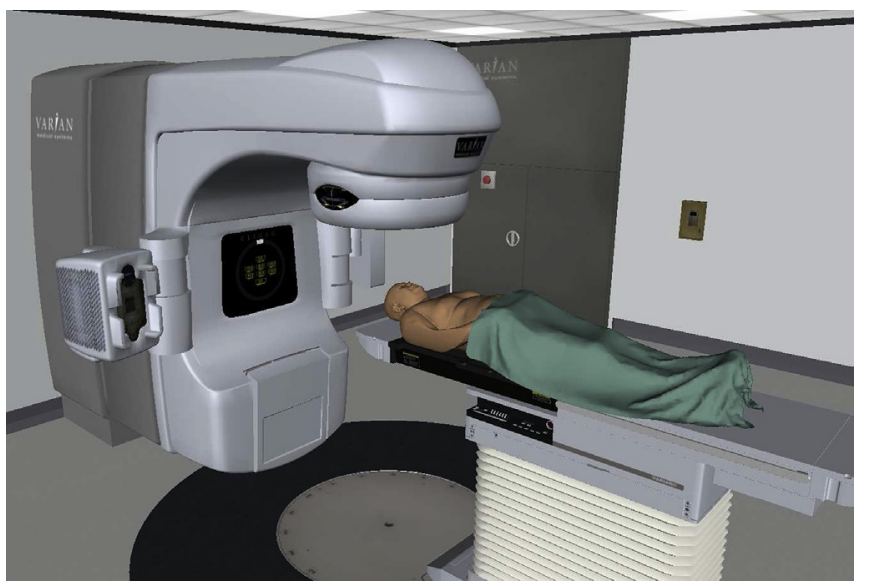

Figure 1. VERT image of a linear accelerator, couch and a virtual patient (courtesy of vertual). can considerably enhance the understanding by patients and their relatives as they can see in the same visual space of the treatment room, the patient, the Linac, the treatment beam, various views of the anatomy of the patient on the treatment couch such as segmented anatomy of the tumour and organs at risk (Fig. 2) and planning data sets. They can also see numerous visualizations of radiation dose distributions such as isodose surfaces, and dose colourwash on surface of tumour and surrounding organs. Finally, VERT also provides a number of training tools that help the doctor to explain specific concepts in RT to patients, such as the isocentre concept and automated placement of skin tattoo marks on the virtual patient.

Our aim was not only to understand what are the needs of patients during the whole process from planning the RT up to treatment delivery but more important, to assess whether this exercise increases patients and relatives' knowledge of RT planning and delivery. To the best of our knowledge, this pilot study involving patients with different types of cancer is the first study on a one-to-one basis and using patients' own CT Scans in which relatives were also involved in the provision of information using VR.

\section{Patients and methods}

\section{Study design}

One hundred and fifty two patients with the histological diagnosis of cancer were asked to participate in this study. Two patients declined to participate in the study as they thought that enough information had already been provided to them. Therefore, 150 patients have been included in this study. The study was open to patients undergoing RT with curative intent and to patients with all types of cancer. A limitation was the time between completing the CT planning for RT and the start of RT. In several cases this period of time was so short to the point that it was not possible to show patients how RT is planned and given using this methodology prior to starting RT. This was specially the case for patients with gynaecological cancers. This was not an issue for patients with breast, prostate, rectum and lung cancer. This study was carried out over a period of 2 years. This research was approved by the human research ethics committee of West Midlands - Staffordshire. Patients were identified by an oncologist who explained to them the aims of the study and what it entailed. Patients were given an

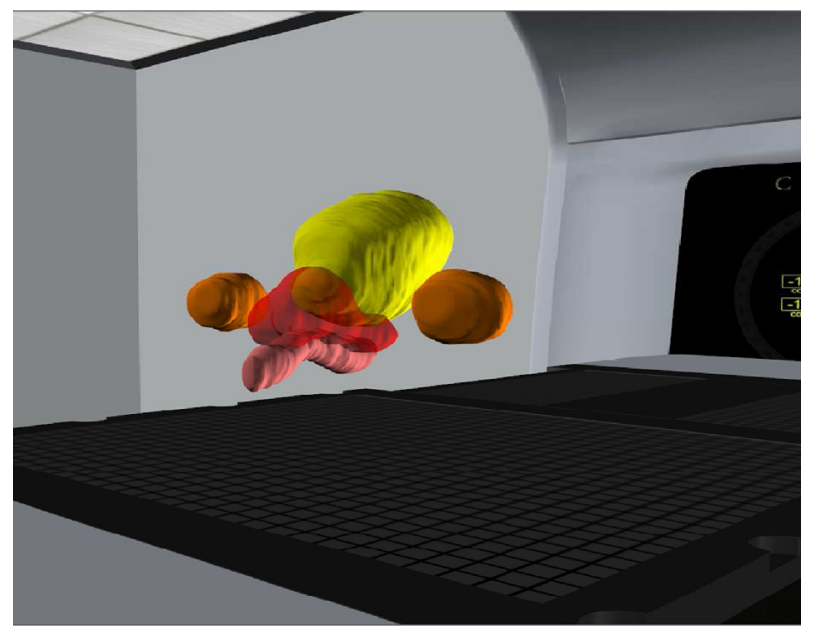

Figure 2. VERT image showing the bladder (yellow), rectum (pink), both femora heads (orange) and the PTV (red) for a patient receiving RT for a cancer of the prostate. 
information sheet and allowed $24 \mathrm{~h}$ to take a decision and before signing a consent form. Patients' information has been summarised in Table 1.

For each study patient, their CT Scan images and RT plan were uploaded onto the VERT system using the Digital Imaging and Communication in Medicine (DICOM) standard which has been developed to enable standardised storage and transfer of medical images with associated RT planning detail overlay. ${ }^{17}$ This means that a database for each patient can be created.

Patients and their relatives were taken to a room and shown using the VERT 3D imaging system how RT is planned and given. Patients and relatives were asked to wear 3D glasses. The information was given on a one-to-one basis involving the patient and his/her relative and/or friend with an oncologist or a radiographer. Patients and relatives were shown first a virtual image of a room were RT is given and the virtual image of a linear accelerator and a couch including the different movements supported by both. Then, they were shown their own CT Scan with emphasis put on the area to be treated and the organs around it. Using this virtual imaging, the Clinical Target Volume (CTV), Planning Target Volume (PTV) and the isocentre concepts were explained. Finally, the pathway of each individual beam was shown and used to explain in more detail possible side effects. At the end of the exercise, patients were asked to fill in a questionnaire to assess their needs. The questionnaire comprised of 8 items. Seven of these were statements relating to tumour visualisation and RT planning. Examples included "What was your level of need for being able to see your own CT Scan images?", "What was your level of need for being able to discuss how RT planning affects side effects?" These statements were accompanied by responses ranked on a Likert scale from 1 to 4: 1 was "not applicable", 2 was "low need", 3 was "moderate need" and 4 was "high need". The eighth and final question invited any other comments or observations wherein participants could insert free text if they so wished.

The uploading of the CT scan images onto the VERT 3D system took around $10 \mathrm{~min}$ for each individual patient. The time needed to explain using VR how RT is planned and given and answer patients and relatives' questions took around $30 \mathrm{~min}$. Patients were asked to fill in the questionnaire at home after the VERT 3D session and bring the questionnaires back in their following visit to the Cancer Centre.

One of the issues raised prior to this pilot study was the setting up of a control group. Initially, patients were asked whether they would be happy being randomised to a control group in which the information on RT planning and delivery would be explained in 2D using their own CT Scans but without using the VERT system. The initial comments were that they would prefer seeing their RT planning in 3D using the VERT system. Therefore, based on patients' comments, a control group in which patients would be shown only the RT planning and delivery in 2D was not included.

Table 1

Summary of study patients' details.

\begin{tabular}{ll}
\hline $\begin{array}{l}\text { Number of patients } \\
\text { Males }\end{array}$ & 95 \\
Females & 55 \\
Age & \\
Total, mean (range) & $68(37-80)$ \\
Males, mean (range) & $68(49-80)$ \\
Females, mean (range) & $58(37-78)$ \\
Histological diagnosis & \\
Prostate & 90 \\
Breast & 52 \\
Rectum & 4 \\
Lung & 3 \\
Thymus & 1 \\
\hline
\end{tabular}

\section{Eligibility and exclusion criteria}

Patients with the histological diagnosis of cancer attending the Cancer Centre for RT were included in this pilot study. All patients had a planning CT Scan as part of the standard radiotherapy process and no further CT Scans were required. The only exclusion criteria was patients unable to fully understand the nature of the study and therefore, unable to provide a signed consent form.

\section{Radiotherapy}

Radiotherapy was performed using 10 or 6 MV photons delivered from a Varian 2100IX linear accelerator. 3D treatment planning was performed on the 'Eclipse' system using CT scans.

Prostate cancer patients. Four patients received RT using a three-field multi-leaf (MLC) conformal technique with anterior and two wedged lateral fields. The remaining 86 patients received RT using five-field Intensity Modulated RadioTherapy (IMRT) configuration. ${ }^{18}$ Eighty-nine patients received a total dose of $74 \mathrm{~Gy}$ in 37 fractions to the reference point (isocentre) delivered 5 days a week for 8 weeks. One patient with recurrent prostate cancer who had undergone prostatectomy 7 years before received a total dose of $66 \mathrm{~Gy}$ in 33 fractions.

Breast cancer patients. Radiotherapy was given using a tangential pair set up. Seven patients received a total dose of $27 \mathrm{~Gy}$ in 5 fractions and a boost of $10 \mathrm{~Gy}$ in 5 fractions as part of the FASTForward study. Four patients received a total dose of $40.05 \mathrm{~Gy}$ in 15 fractions and a boost of $16 \mathrm{~Gy}$ in 8 fractions as part of the IMPORT HIGH study. Fourty-one patients received a total dose of $40.05 \mathrm{~Gy}$ in 15 fractions. Twelve patients also received radiotherapy to the supraclavicular fossa to a total dose of $40.05 \mathrm{~Gy}$ in 15 fractions.

Lung cancer patients. Radiotherapy was given with a 3 field CT planned volume. Two patients received a total dose of $55 \mathrm{~Gy}$ in 20 fractions. One patient received a total dose of $30 \mathrm{~Gy}$ in 10 fractions.

Rectal cancer patients. Radiotherapy for rectal cancer was given with a 3 field conformal planned volume. All 4 patients received a total dose of $45 \mathrm{~Gy}$ in 25 fractions.

Thymoma patient. Radiotherapy was given using a conformal volume. The patient received $50 \mathrm{~Gy}$ in 20 fractions.

All patients completed the prescribed dose of RT.

\section{Data analysis}

Descriptive analysis (frequencies and percentages) was generated from the 7 rated response items in the questionnaire. This yielded the proportion of patients who had a moderate or high need for better understanding how RT is planned and delivered. Following descriptive analysis, a content analysis was performed upon all of the responses to the free text components of the questionnaire. The free text open ended comments were collated and themes were established. This involved reading all of the responses as a set of data and then coding the data into closed categories, such as "information and understanding", "efficacy of visual information" and "emotional factors". Appropriate extracts were assigned to relevant codes, and the data were summarised accordingly. However, these have not been presented under headings as the data obtained was deemed not sufficient to warrant this. Although interviews of focus groups yield data for this sort of presentation, in this case, these were short comments at the end of the questionnaire that were categorised. It was a simple exercise of looking for thematic coherence amongst the responses at a descriptive level and the main purpose was to add to the descriptive data presented from the other questions. 


\section{Results}

One hundred and four out of 150 patients (69.3\%) received information on RT planning and delivery using VR with their partners. Four $(2.6 \%)$ patients attended with a friend. Three $(2.0 \%)$ patients attended with their daughter. One patient attended this exercise with both his partner and his daughter, another patient attended with his partner and 2 sons, and another one attended with her mother. Thirty five (23.3\%) patients received the information alone.

The analysis of patients' needs during the whole process of RT planning and delivery showed that $83.0 \%$ of patients had a moderate or high need to better understand using 3D imaging how RT is planned, and $83.3 \%$ of patients had a moderate or high need to understand using 3D imaging how RT is delivered. Furthermore, 80.6\% of patients had a moderate or high need to see the area to be treated using their own CT Scans uploaded onto the VERT 3D system.

Of the 150 participants who completed the questionnaire, 87 responses (58\%) to the open ended question that invited them to add any additional comments about the RT planning exercise were screened to establish the prevalent positive and negative issues raised. All respondents cited greater understanding as a positive outcome of the exercise. Understanding fell into different contexts. The most cited context was improved understanding about the nature of prostate cancer, which generally added to the knowledge that individuals had already gained throughout the diagnostic process.

On the other hand, procedural understanding was deemed to have been greatly improved with many respondents explicitly stating that they were better informed about how treatment would be performed. While this observation mostly focussed upon patients' own procedural understanding, others also extended this to improving knowledge for relatives about the technical delivery of treatment, with one response actually describing it as "a good experience for both patient and family". A more specific facet of understanding related to procedural issues was also shown within patients' responses. This pertained to side effects and demonstrated that while participants knew that they would experience side effects, the RT planning exercise helped them to anticipate side effects more, as the following response exemplifies:

"I now have a good working knowledge of what my treatment involves and a better understanding of any side effects that may occur".

Reponses that pointed to improved understanding on different levels also linked closely with emotional aspects that were inherent to the RT exercise. Participants commented that their improved understanding on different levels contributed to a reduction in anxiety and the "fear factor". For example:

"This is an excellent opportunity to become involved with the treatment and a greater understanding helps to remove the "fear factor" and helps in the healing process".

Additionally, they also referred to the RT planning exercise as reassuring. This could admittedly be argued as something that would be present in all types of consultations and something that is largely dependent on other factors, such as the communication skills of the healthcare professional, and therefore leads to the raison d'être of the exercise - the perceived efficacy of visual information.

There was a marked inclusion of comments that highlighted the usefulness of the ability to see a visual representation of the area being treated. For example:
"A picture is more powerful to assist understanding than many words, especially to show the size and shape in relation to other organs".

This seems to be a large contributory factor in assisting all the other positive outcomes associated with greater understanding and anxiety reduction. For one participant the experience facilitated a more "meaningful" discussion with the health professional, thereby implying that meaning and understanding might otherwise be diminished without the additional benefits of visual representations. This also might indicate that patients would cite improved communication between themselves and clinicians more frequently.

However, while participants mainly spoke of their own thoughts and feelings about the exercise with relation to their specific cases, it was also interesting to note the inclusion of collective interest in responses. Several extended to asserting how the imaging technology would be beneficial to assisting other patients as well as its positive impact on individual experiences:

"I think carrying out this exercise before RT starts would be beneficial and reassuring to most patients."

The common denominator in this study was that all patients and relatives welcomed this exercise as it helped them to better understand how RT is planned and delivered and why they might get certain side effects. The aim of this study was to assess whether this extra exercise increases patients and relatives' knowledge of RT planning and delivery. Based on the data presented here and the statements provided by several patients, we believe that this extra exercise increases both patients and relatives knowledge on RT planning and delivery. Furthermore, from the patients' point of view, the possibility of seeing the room where RT is given and the Linac delivering it before starting RT reduced their anxieties when entering it for the first time. For relatives, the possibility given to them to see also this room made them feel more involved in the treatment of their loved ones as, in general, they do not have the opportunity to enter it.

Another important point raised by both patients and relatives was the more relaxed atmosphere when receiving this information in $3 \mathrm{D}$. Both patients and relatives stated that this experience was in some instants similar to being in the cinema watching a 3D movie, something that is generally associated with enjoyment. The VERT system also has a 2D license. However, the patients and relatives' comments in this case strengthen the case for delivering information on RT planning and delivery using a 3D imaging system.

\section{Discussion}

Radiotherapy is one of the main treatments for cancer and RT planning a critical component in the safe and effective delivery of this treatment. Also, the improvements in RT planning and delivery together with an increased life expectancy are leading to a predicted rise in the use of RT in the UK. ${ }^{14}$

The way RT is planned is usually explained to patients using CT Scan imaging in 2D. However RT is difficult for patients to comprehend because it is not visible. ${ }^{19}$ The provision of information to patients receiving RT and their relatives using a 2D imaging system such as CT Scans could be improved using VR. It is well recognised that spatial relationships are easier to understand when visualized stereoscopically in 3D environments, and VR systems have therefore the potential to improve the RT planning process ${ }^{17}$ and even provide images of the room where RT is given and the machine delivering it. ${ }^{11}$

This pilot study was carried out to assess whether providing information on RT planning and delivery using VR increases 
patients and relatives knowledge on RT planning and delivery. While it is accepted that 3D imaging is better than 2D imaging to better understand spatial relationships, ${ }^{17,20}$ the work carried out so far using 3D imaging and VR have been aimed mainly at improving RT teaching. ${ }^{13}$

The results obtained in this study highlight that this extra information was welcomed by patients to the point that it was stressed by patients themselves that information using VR on RT planning and delivery should be provided to everybody receiving RT, and before starting treatment. More important, patients and relatives felt that their knowledge on RT planning and delivery had been improved. This strengthens the case for VR as a tool to provide further information on RT planning and delivery. Also, from the relatives' point of view, another main message from them was that they now felt more involved in the treatment of their loved ones. This was achieved by being able to see not only the room where RT is given and the machine delivering but also to see and understand all the steps taken inside the RT room in order to set up the patient before RT is delivered, and how the treatment is given. However, it is important to recognise that not all patients wished to receive this information using a VR system as two of them thought that they had already been fully informed. Furthermore, some partners did not wish to attend this exercise as they felt also enough information had been given to them.

On the other hand, we believe that providing information on RT planning and delivery using a 3D imaging system could be easily applied in Oncology departments. The whole time to upload the CT Scans and explaining patients and relatives how RT is planned and given using this system should, in our experience, not take more than $40 \mathrm{~min}$. Apart from the capital cost of purchasing the VERT system (including licences and software update), the only additional expense in this study was the time for an oncologist or a radiographer to explain to patients how RT is planned and given using VR. We were able to provide 3 sessions a week and always during one of the patients' visit to the Cancer Centre. This number of sessions was based on the allocated time an oncologist and several radiographers at the Cancer Centre had for this exercise. Obviously, this could vary from one oncology department to another. Furthermore, all sessions were carried out during the 9.00 till 5.00 working hours. However, other centres might also be able to offer evening sessions.

Another issue concerns the use of the patient's own CT Scans. It could be argued that a more economic way to give this information would be to use a standard anonymised CT Scan and showing the planning and delivery of RT to several patients and their relatives at the same time. However, from this pilot study, we believe this would reduce the value of the demonstration compared to a oneto-one presentation for two reasons. Firstly, patients usually asked whether this information could be given using their own CT Scans. In fact, in one case with a patient with prostate cancer with an artificial hip, he wished to see his own CT Scan as the RT planning was different to patients without an artificial hip (avoiding the artificial hip when delivering RT). Secondly, in general, patients and their relatives might feel more at ease when asking questions to a doctor on a one-to-one basis rather than in the presence of other patients and relatives. Therefore, we believe that provision of information using a VR imaging system should use whenever possible the patient's own CT Scan.

\section{Conclusions}

The effective provision of information on RT planning and delivery for patients with cancer is a vital issue in improving patients' satisfaction. Providing this information using 3D imaging systems rather than $2 \mathrm{D}$ helped patients and relatives to better understand the different steps in RT planning and reduced their anxieties. This study shows for the first time that both patients and relatives would welcome information on RT planning and delivery using a 3D imaging system on a one-to-one basis with an oncologist. This work strengthens the case for such a system to be established in Oncology departments.

\section{Conflict of interest}

None.

\section{Acknowledgements}

The authors would like to thank the radiographers at the Cancer Centre, and the staff at the Medical Physics Department, Cancer Centre, UHNS, specially Dr C. Edwards and Miss A. Patel, for their help in this study. The input from Mrs J. Antons (VERTUAL) is also acknowledged. Funding from the Cancer Centre (UHNS), the Dott Griffiths Cancer Appeal Fund, and the Rotary Clubs of Blythe Bridge and District, Newcastle under Lyme, and Tunstall is also acknowledged.

\section{References}

1. Wagner EH, Bowles EJA, Greene SM, Tuzzio L, Wiese CJ, Kirlin B, et al. The quality of cancer patient experience: perspectives of patients, family members, providers and experts. Qual Saf Health Care 2010;19:484-9.

2. Fallowfield L, Jenkins V. Effective communication skills are the key to good cancer care. Eur J Cancer 2001;35:1592-7.

3. Meredith C, Symonds P, Webster L. Information needs of cancer patients in west Scotland: cross sectional survey of patients' views. BMJ 1996;313:724-6.

4. Passalacqua R, Caminiti C, Campione F, Diodati F, Todeschini R, Bisagni G, et al. Prospective, multicenter, randomized trial of a new organizational modality for providing information and support to cancer patients. J Clin Oncol 2009;27: 1794-9.

5. Department of Health. The NHS cancer plan: education and support for district and community nurses in the principles and practice of palliative care education. 2001. http://www.dh.gov.uk/en/Publicationsandstatistics/Publications/ PublicationsPolicyAndGuidance/DH_4008564.

6. Miller SM. Monitoring versus blunting styles of coping with cancer influence the information patients want and needs about their disease - implications for cancer screening and management. Cancer 1995;76:167-77.

7. Bungay H, Cappello R. As long as the doctors know what they are doing': trust or ambivalence about patient information among elderly men with prostate cancer. Eur J Cancer Care 2009;18:470-6.

8. Pitceathly C, McGuire P. The psychological impact of cancer on patients' partners and other key relatives: a review. Eur J Cancer 2003;39:1517-24.

9. Derdiarian A. Effects of information on recently diagnosed cancer patients' and spouses' satisfaction with care. Cancer Nurs 1989;12:285-92.

10. Phillips R, Ward JW, Beavis AW. Immersive visualization training of radiotherapy treatment. Stud Health Technol Inf 2005;111:390-6.

11. VERTUAL: www.vertual.co.uk.

12. Hubbold RJ, Hancock DJ, Moore CJ. Autosteroscopic display for radiotherapy planning. In: Scott SF, Merritt JO, Bolas MT, editors. Stereoscopic display and virtual reality system IV. SPIE Proceedings, vol. 3012; 1997. p. 16-27.

13. Boejen A, Grau C. Virtual reality in radiation therapy training. Surg Oncol 2011;20:185-8.

14. Report to Ministers from National Radiotherapy Advisory Group (NRAG). Radiotherapy: developing a world class service for England. May 2007.

15. Bridge P, Appleyard RM, Ward J, Phillips R, Beavis AW. Development and evaluation of a virtual radiotherapy treatment machine using an immersive visualization environment. Comput Educ 2007;49:481-94.

16. Philips R, Ward JW, Page L, Grau C, Boejen A, Hall J, et al. Virtual reality training for radiotherapy becomes a reality. Proceedings of medicine meets virtual reality 16. Stud Health Technol Inform 2008;132:366-71.

17. Patel D, Muren LP, Mehus A, Kvinnsland Y, Ulvang DM, Villanger KP. A virtual reality solution for evaluation of radiotherapy plans. Radiother Oncol 2007;82: 218-21.

18. Yu CX, Amies CJ, Svatos M. Planning and delivery of intensity-modulated radiation therapy. Med Phys 2008;35:5233-41.

19. Schafer C, Herbst M. Ethical aspects of patient information in radiation oncology. An introduction and a review of the literature. Strahlenther Onkol 2003; 179:431-40.

20. Su TS, Sung WH, Jiang CF, Sun SP, Wu CJ. The development of a VR-based treatment planning system for oncology. Department of Biomedical Engineering, I-Shou University, Kaohsiung County, Taiwan Conf Proc IEEE Eng Med Biol Soc 2005;6:6104-7. 